\title{
Existence conditions and drift velocities of adiabatic flame-balls in weak gravity fields
}

\author{
Guy Joulin \\ Vadim N Kurdyumov
}

Amable Liñàn

\begin{abstract}
Combining activation energy asymptotics, suitable scalings and numerical methods, we study how flame-balls move under the action of the free convection that they themselves generate in the presence of a weak, uniform gravity field. Attention is focused on steady configurations (in a suitable reference frame), on an isolated flame-ball of size comparable to what is obtained in the absence of gravity, and on deficient reactants that are characterized by a low Lewis number. For the sake of simplicity, we consider an adiabatic combustion process, in the sense that the radiative exchanges are neglected. This work provides one with:

(a) a description of the free-convection field around the flame-ball, along with an asymptotic estimate of the drift velocity;

(b) a relationship between the flame-ball radius, strength of gravity and physico-chemical properties of the reactive premixture;

(c) extinction conditions, caused by the net effect of heat extraction from the flame-ball to its surroundings by the free-convection field.

Hints on generalizations currently under consideration are also given.
\end{abstract}

\section{Introduction}

The early experiments of Coward and Brinsley (1914) revealed that combustion of lean premixtures of such light fuels as hydrogen $\left(\mathrm{H}_{2}\right)$ in air is very peculiar indeed, especially close to the flammability limits. Downward flame propagations cease to be viable when the mole fraction $X_{\mathrm{H}_{2}}$ of $\mathrm{H}_{2}$ drops below about $9 \times 10^{-2}$, whereas some significant 'upward' combustion activity persists until $X_{\mathrm{H}_{2}} \cong 3.5 \times 10^{-2}$. The very form in which combustion manifests itself in the latter situation, namely a few globules or 'balls' moving upward instead of a continuous flame front, as well as later experiments with deuterium instead of $\mathrm{H}_{2}$ (Clusius 1950), suggested that the high mobility of the limiting reactant is responsible for the aforementioned peculiarities (Zel'dovich 1944). Yet no mathematically self-consistent analysis of the motion of flame-balls in a prescribed gravity field has been offered to dateł. Beside the difficulty of the required mathematical analysis, the lack of insight into the gravity/flammability-limit interactions possibly comes from the fact that gravity is not a parameter one can easily tune experimentally. 


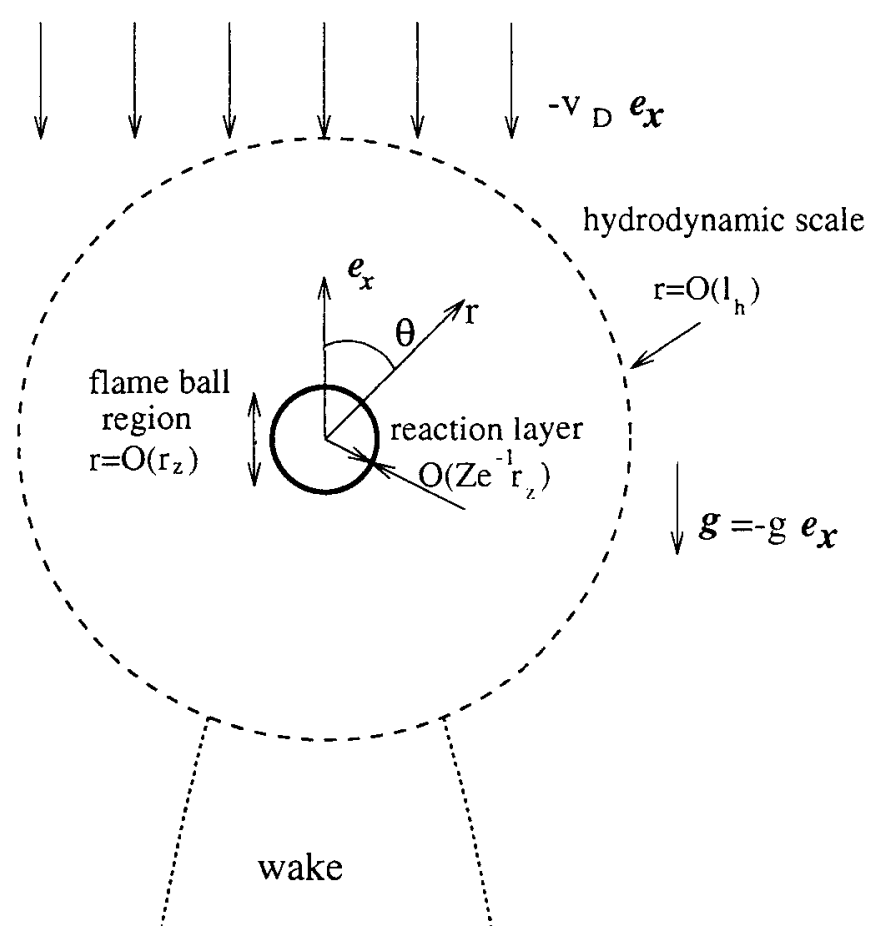

Figure 1. Sketch of the various zones involved in the studied configuration when $Z e \rightarrow \infty$, $\ell_{h} / r_{Z} \rightarrow \infty$ and $r_{F} / r_{Z}=\mathrm{O}(1)$.

This has changed recently, since experiments at reduced gravity have become feasible (Ronney et al 1998), e.g. during orbital flights.

The present work offers the simplest mathematically consistent description of a single localized combustion spot (cf figure 1) of the flame-ball type (Zel'dovich et al 1985) that rises at constant velocity as a result of the free-convection flow field it itself generates when a uniform, weak gravity field acts on it. In addition to providing one with non-trivial results, the advantage of considering weak gravity fields is that they lead to scale separation between two main regions (figure 1), namely

- A flame-ball region, dominated by chemistry and diffusive processes, and of size comparable to the flame-ball radius $\left(r_{Z}\right)$ identified by Zel'dovich (1944); when evaluated in a frame attached to the ball, convection is insignificant there when gravity is small, and the combustion process is spherically symmetric up to the relevant leading orders.

- A wider, nearly isothermal far field; its size $\left(\ell_{h}\right)$ is determined by the rate at which the far field receives heat from the flame-ball and by gravity, through the requirement that conduction and buoyancy-induced convection balance one another. The shifts in temperature and reactant concentration the far-field flow induces about the flame-ball are small, $\mathrm{O}(1 / \mathrm{Z} e$ ), but non-negligible when the Zel'dovich number $Z e$ is large (as is assumed here) and when a suitable distinguished limit between $Z e$ and gravity (equation (3.5)) is considered.

Handling both regions separately (analytically for the first one and numerically for the second) then invoking matching will ultimately yield the flame-ball size and its drift velocity as functions of gravity and mixture properties. For the sake of simplicity we only consider 
adiabatic configurations, in the sense that no volumetric heat-loss (e.g. radiant) term is accounted for in the energy balance, equation (2.2); admittedly this is an important limitation, which shall need be relaxed before comparisons with experiments are attempted. Studying the various roles of radiative exchanges will be contemplated in a forthcoming work.

The present paper is organized as follows. In section 2 , the mathematical model is defined. The Zel'dovich basic solution, its simple generalizations and the chosen ordering are then presented (section 3 ). The outer hydrodynamical problem is treated in section 4 and the flameball radius, then its drift velocity, are determined in section 5 . We offer concluding remarks in section 6 .

\section{Mathematical model}

We envisage situations where the flame-ball rises through an infinite medium of density $\rho_{u}$ and temperature $T_{u}$, at a constant speed $v_{D}$ in a ground-based frame of reference. It is then appropriate to formulate the problem in a reference frame rising with the flame-ball, in which case the whole configuration may be considered as steady state. The continuity (2.1) and energy (2.2) equations, and the balance (2.3) for the deficient reactant, are then written as follows:

$$
\begin{aligned}
& \nabla \cdot(\rho v)=0 \\
& c \rho v \cdot \nabla T=\nabla \cdot(\lambda \nabla T)+Q w \\
& \rho v \cdot \nabla y=\nabla \cdot(\rho D \nabla y)-w
\end{aligned}
$$

where $\rho$ (mixture density), $T$ (local temperature), $v$ (mixture velocity vector), $\lambda$ (heat conductivity), $c$ (specific heat at constant pressure), $D$ (diffusion coefficient of limiting reactant) and $y$ (reactant mass fraction) have their standard meanings; for the sake of simplicity both $\lambda\left(\equiv \lambda_{u}\right.$ ) and $\rho D$ will be considered to be constant; also, we shall neglect the changes in molar weight caused by non-uniform composition, and the approximation $\rho T=\rho_{u} T_{u}$ is adopted for the small-Mach-number flows we study here. $Q$ denotes the heat of reaction per unit mass of deficient reactant, and $w$ represents the rate at which the latter is consumed. In equations (2.1)-(2.3) we explicitly make the assumption that the combustion process under consideration is modelled by an irreversible reaction 'fuel $\rightarrow$ products + heat'. The reaction rate $w$ is selected to be of Arrhenius type:

$$
w=\rho y \mathrm{e}^{-T_{a} / T} / t_{c}
$$

where $T_{a}$ is the activation temperature and $t_{c}$ stands for a reference 'collision' time.

The velocity $v$ relative to the flame-ball involved in (2.1)-(2.3) follows from the steady, variable-density version of the Navier-Stokes equations, namely

$$
\rho(v \nabla) \cdot v=-\nabla p+\mu_{u} \nabla^{2} v-\left(\rho-\rho_{u}\right) g e_{x} .
$$

In equation (2.5) $p$ represents the pressure changes due to flow motion (the ambient hydrostatic contribution corresponding to $\rho \equiv \rho_{u}$ has been subtracted); $\mu_{u}$ is the ordinary viscosity of the fresh medium; $g>0$ denotes the acceleration of gravity and $e_{x}$ is the unit vector in the 'up' direction defined by it. Because equation (2.5) is to be effectively used in regions where the temperature (and hence density) is nearly uniform, $\mu_{u}$ was assumed to be constant in (2.5).

The boundary conditions associated with $(2.1)-(2.3)$ require that

$$
T \rightarrow T_{u} \quad y \rightarrow y_{u}
$$

at large enough distances $(r=|r| \rightarrow \infty)$ from the combustion spot. As for the velocity field $v$, we require that

$$
v \rightarrow v(\infty)=-v_{D} \boldsymbol{e}_{x} \quad \text { for } \quad \boldsymbol{r} \rightarrow \infty
$$


at least if $\boldsymbol{r}$ lies outside of the wake (figure 1) which the ascending flame-ball leaves behind; how to handle the wake itself will be explained in section 4. As expressed in (2.7) the flame-ball drifts upward, with a velocity $v_{D}>0$ which is as yet unknown and constitutes an eigenvalue of the problem at hand. Its value is to be fixed by the condition that the flame-ball would be dragged by the flow. In other words, the flame-ball and the flow locally have a vanishing relative velocity; as shown later on, this effectively imposes that

$$
\boldsymbol{v}(\boldsymbol{r}=\mathbf{0}) \cong \mathbf{0}
$$

or, better, that $v\left(r=\mathrm{O}\left(r_{F}\right)\right)$ is negligible compared to the typical, $\mathrm{O}\left(v_{D}\right)$, velocities prevailing in the region where (2.1) and (2.5) must be accounted for to leading order (see section 6).

Our task from now on is to compute the profiles of $T, y, v$ (including $v_{D}$ ) and to determine the conditions under which equations (2.1)-(2.8) have solutions.

\section{Zel'dovich solution; orders of magnitude}

In the absence of any convection $(g=0, \boldsymbol{v} \equiv \mathbf{0})$ equations (2.1)-(2.3) possess spherically symmetric, 'Zel'dovich' solutions for which $T, y, \rho \sim 1 / T$ only depend on the distance $(r)$ from the origin $\boldsymbol{r}=\mathbf{0}$. These so-called flame-balls (Zel'dovich 1944) admit the first integral $T+Q y / c L e \equiv$ constant, where $L e=\lambda / \rho c D$ is the Lewis number of the deficient reactant. Once the above integration constant $\left(=T_{u}+Q y_{u} / c L e\right)$ is evaluated far from the flame-ball where $T=T_{u}, y=y_{u}$, setting $y=0$ in the first-integral defines the reference reaction temperature

$$
T_{*}=T_{u}+\left(T_{b}-T_{u}\right) / L e
$$

where $T_{b} \equiv T_{u}+Q y_{u} / c$ represents the temperature attained at the burned side of a flat flame propagating in a fresh medium characterized by $T=T_{u}, y=y_{u}$. Such highly mobile deficient reactants as $\mathrm{H}_{2}$ have $L e<1$ and hence $T_{*}>T_{b}$.

In the limit of large Zel'dovich numbers, $Z e \equiv T_{a}\left(T_{*}-T_{u}\right) / T_{*}^{2} \rightarrow \infty$, the chemical term $w$ featured in (2.2) and (2.3) is non-negligible only in a thin shell which is spherical when $\boldsymbol{v} \equiv \mathbf{0}$, of radius $r_{Z}$, and has a thickness $\mathrm{O}\left(r_{Z} / Z e\right)$. Standard asymptotic methods (Buckmaster and Ludford 1982) yield the leading-order result (Joulin 1985)

$$
r_{Z}^{2}=\frac{1}{2} D\left(T_{R}\right) t_{c} \mathrm{e}^{T_{a} / T_{R}}\left(T_{a}\left(T_{b}-T_{u}\right) / T_{R}^{2}\right)^{2}
$$

with, here, $T_{R} \equiv T_{*}$. If the values of $T$ and $y$ far from the flame-ball are shifted by $\delta T_{\infty}$ and $\delta y_{\infty}$ from their nominal values $T_{u}$ and $y_{u}$, equation (3.1) predicts a shift in reaction temperature $T_{R}-T_{*} \equiv \delta T_{R}$ given by

$$
\delta T_{R}=\delta T_{\infty}+\left(T_{*}-T_{u}\right) \delta y_{\infty} / y_{u}
$$

The corresponding change in flame-ball radius is from $r_{Z}$ to $r_{F}$, with

$$
\frac{r_{F}}{r_{Z}}=\exp \left(-\frac{T_{a}}{T_{*}} \frac{\delta T_{R}}{2 T_{*}}\right)
$$

to leading order in the limit $Z e \rightarrow \infty$.

Interestingly enough, deriving the above results (3.1)-(3.4) only required that the flame structure at the scale $r=\mathrm{O}\left(r_{Z}\right)$ be considered as steady and convection-free up to $\mathrm{O}\left(Z e^{-2}\right)$ and also that the fields of $y$ and $T$ at $r \gg r_{F}$ be isotropic to the same accuracy. Accordingly, equations (3.3) and (3.4) will still hold when the above evoked $\delta T_{\infty}$ and $\delta y_{\infty}$ result from unsteady and/or convective phenomena that happen to be non-negligible only when $r \gg r_{F}=$ $\mathrm{O}\left(r_{Z}\right)$, and provided the aforementioned condition of isotropy is fulfilled. This has been exploited previously to set up simple models of flame initiation by a heat source (Joulin 1985) 
or to study the response of flame-balls to velocity gradients and volumetric heat losses (see Buckmaster and Joulin 1991 and references therein). The same approach is adopted here, $\delta T_{\infty}$ and $\delta y_{\infty}$ are now due to the free-convection field which the flame-ball itself generates at $r \gg r_{F}$ when $g$ is small but non-zero.

The constraint that $\delta T_{\infty} / T_{*}$ and $\delta y_{\infty} / y_{u}$ be $\mathrm{O}(1 / Z e)$, as required by (3.4) for $r_{F} / r_{Z}$ to be $\mathrm{O}(1)$ when $\mathrm{Z} e \rightarrow \infty$, puts bounds on the intensity of gravity. As shown in the next section this will require that

$$
g r_{Z}^{3} /\left(\lambda / \rho_{u} c\right)^{2} \sim Z e^{-2}
$$

The quantity on the left-hand side of (3.5) is a Grashof number based upon $g, r_{Z}$ and thermal diffusivity $\left(\lambda / \rho_{u} c\right)$. The condition (3.5) will imply that the length scale $\left(\ell_{h}\right)$ over which convection becomes important satisfies

$$
\ell_{h} \sim \operatorname{Zer}_{Z}
$$

thereby ensuring scale separation between the flame-ball and the surrounding hydrodynamical field.

Before moving to the hydrodynamical scale proper, a remark is in order: provided $\delta T_{\infty} \ll T_{*}, \delta y_{\infty} \ll y_{u}$ and the flame-ball structure corresponding to $r=\mathrm{O}\left(r_{Z}\right)$ may effectively be considered as spherically symmetric (up to $\mathrm{O}\left(\mathrm{Ze}^{-2}\right)$ corrections), the profiles of $T$ and $y$ prevailing for $r \geqslant r_{F}$ will satisfy

$$
\begin{aligned}
& T-T_{u}=\left(T_{*}-T_{u}\right) r_{F} / r+\delta T_{\infty}+\cdots \\
& y=y_{u}\left(1-r_{F} / r\right)+\delta y_{\infty}+\cdots
\end{aligned}
$$

when $r_{Z} \ll r \ll \mathrm{O}\left(Z e r_{Z}\right)$. In other words, the flame-ball structure evaluated in terms of the physics pertaining to the length scale $r=\mathrm{O}\left(r_{F}\right)$ acts (in a first approximation) as a point-source of heat and a point sink of reactant when seen from a distance $r \gg r_{F}$. The corresponding strengths are $q \equiv 4 \pi \lambda r_{F}\left(T_{*}-T_{u}\right)$ and $-4 \pi y_{u} \rho D r_{F}$, respectively. The as yet unknown $\delta T_{\infty}$ and $\delta y_{\infty}$ will be determined by analysing the hydrodynamical region $\left(r=\mathrm{O}\left(\ell_{h}\right)\right)$, through a two-term matching with (3.7).

\section{Hydrodynamical problem}

As mentioned above, the flame-ball, of as yet unknown radius $r_{F}$, acts at a point source/sink of heat/reactant when seen from a distance $r \gg r_{Z}$. To compute $r_{F} / r_{Z}$ from (3.4), four main problems need to be elucidated: (a) find the length, velocity, temperature and mass-fraction scales corresponding to the region where convection is significant; (b) check that $v$ is indeed negligible when $r=\mathrm{O}\left(r_{Z}\right)$ as well as the legitimacy of (2.8); (c) solve (2.2) and (2.5) for $T$, $y, v$ in the hydrodynamic zone surrounding the flame-ball, with the apparent shifts $\delta T_{\infty}$ and $\delta y_{\infty}$ in 'ambient' conditions as by-products; (d) plug the results into (3.4) and find $r_{F}$ itself, along with $v_{D}$.

\subsection{Scales}

As first shown by Zel'dovich (1937), and more recently exploited by Kurdyumov and Liñàn (1999) (see also Hieber and Gebhart 1969), the typical length scale $\left(\ell_{h}\right)$, velocity scale $\left(v_{h}\right)$ and temperature variations $\left(T_{h}-T_{u}\right)$ pertaining to the free-convection field around a point heat source of given power $q$ are determined by the requirements that heat conduction, inertia and buoyancy would balance one another. Accordingly, one requires that

$$
\left(T_{h} / T_{u}-1\right) g \ell_{h} \sim v_{h}^{2} \quad v_{h} \ell_{h} \sim \lambda_{u} / \rho_{u} c \quad\left(T_{h}-T_{u}\right) \lambda_{u} \ell_{h} \sim q
$$


which yields (with $D_{t h} \equiv \lambda_{u} / \rho_{u} c$ )

$\ell_{h}=\left(D_{t h}^{2} \lambda_{u} T_{u} / q g\right)^{1 / 2} \quad v_{h}=\left(q g / \lambda_{u} T_{u}\right)^{1 / 2} \quad T_{h}-T_{u}=\left(q^{3} g / T_{u} D_{t h}^{2} \lambda_{u}^{3}\right)^{1 / 2}$.

Reactant diffusion balances convection over the scales defined by (4.2). One can next determine a typical far-field reactant mass fraction $y_{h}-y_{u} \sim y_{u} r_{Z} / \ell_{h}$, once an order-of-magnitude matching with (3.7) is accounted for. We shall define $y_{h}$ by

$$
y_{h}-y_{u}=4 \pi \text { Le } y_{u} r_{F} / \ell_{h} .
$$

As for the value of $q$ itself, equation (3.7) implies

$$
q=4 \pi \lambda_{u} r_{F}\left(T_{*}-T_{u}\right)
$$

\subsection{Velocity on the $O\left(r_{Z}\right)$ length scale}

As is to be found in the next subsection, the following estimate:

$$
v \leqslant \mathrm{O}\left(v_{h} \boldsymbol{r} / \ell_{h}\right)
$$

holds when the hydrodynamical field corresponding to $r=\mathrm{O}\left(\ell_{h}\right)$ is specialized to $r / \ell_{h} \ll 1$. An estimate of $v$ for $r=\mathrm{O}\left(r_{Z}\right)$ is then given (on the grounds of matching) by $v \sim v_{h} r_{Z} / \ell_{h}$; this is $\mathrm{O}\left(Z e^{-2}(\lambda / \rho c) / r_{Z}\right)$ if (3.5) holds, in which case convection is negligible at the flame-ball scale, as assumed to derive (3.4). The problem of isotropy is to be handled below.

\subsection{Free-convection field}

To analyse the flow field on the scales defined by (4.2) and (4.3), we introduce the reduced dependent and independent variables

$$
\begin{aligned}
& \boldsymbol{V}=\boldsymbol{v} / v_{h} \quad \boldsymbol{R}=\boldsymbol{r} / \ell_{h} \\
& \left(T-T_{u}\right)=\left(T_{h}-T_{u}\right) \Theta+\cdots \quad y-y_{u}=-\left(y_{h}-y_{u}\right) Y+\cdots \\
& \rho=\rho_{u}+\cdots
\end{aligned}
$$

which transform the chemistry-free forms of (2.1)-(2.3) and (2.5) into

$$
\begin{aligned}
& \nabla \cdot(\boldsymbol{V})=0 \\
& \boldsymbol{V} \cdot \nabla \Theta=\nabla^{2} \Theta+\delta(\boldsymbol{R}) \\
& \boldsymbol{V} \cdot \nabla Y=\nabla^{2} Y / L e+\delta(\boldsymbol{R}) \\
& (\boldsymbol{V} \cdot \nabla) \boldsymbol{V}=-\nabla P+P_{r} \nabla^{2} \boldsymbol{V}+e_{x} \Theta
\end{aligned}
$$

where the differential operators now refer to the reduced coordinates and $P_{r} \equiv \mu_{u} c / \lambda_{u}$ is the far-field Prandtl number. $P$ represents $p$ in units of $\rho_{u} v_{h}^{2}$. Equations (4.9)-(4.12) are nonlinear Boussinesq equations; no buoyancy caused by changes in composition appears, as we chose $\rho T=\rho_{u} T_{u}$ as the equation of state. The boundary conditions associated with (4.9)-(4.12) include

$$
\Theta \rightarrow 0 \quad \boldsymbol{Y} \rightarrow 0 \quad \text { and } \quad \boldsymbol{V}+V_{D} \boldsymbol{e}_{x} \rightarrow 0 \text { for } \boldsymbol{R} \rightarrow \infty
$$

with $V_{D} \equiv v_{D} / v_{h}$, as follows from (2.6); actually the last condition in (4.13) only holds outside of a slender wake, as explained later on. Due to the presence of concentrated sources in (4.10) and (4.11), the functions $\Theta$ and $Y$ diverge as $R \rightarrow 0$ and to facilitate the numerical resolution, their local behaviour is needed. A coordinate expansion reveals that, provided $V(0)$ satisfies (2.8) (see also (4.23)), $\Theta$ and $Y$ behave as follows $\dagger$ for $R=|R| \rightarrow 0$ :

$$
\Theta \cong \frac{1}{4 \pi|R|}+B(1)+\mathrm{O}(R) \quad Y=\frac{L e}{4 \pi|R|}+B(L e)+\mathrm{O}(R) .
$$

$\dagger$ Otherwise the additional terms $V(\boldsymbol{0}) \cdot e_{x} \cos \vartheta / 8 \pi$ and $V(\boldsymbol{0}) \cdot e_{x} \cos \vartheta L e / 8 \pi$ would appear in the right-hand sides. 
$B(1)$ and $B(L e)$ are as yet unknown numbers, to be determined from the numerical resolution of (4.8)-(4.11). Since the first terms in the right-hand sides of (4.14) are sufficient to specify $\Theta(R)$ and $Y(R)$, it is enough to require that

$$
4 \pi R^{2} \frac{\partial \Theta}{\partial R}=-1 \quad 4 \pi R^{2} \frac{\partial Y}{\partial R}=-L e \quad \text { at } \quad R \rightarrow 0
$$

which does not depend on $B(1), B(L e)$. In the numerics, this was imposed at the surface of a small sphere centred at $R=0$ and with a radius $R_{-}$of $\mathrm{O}\left(10^{-3}\right)$; allowing $R_{-}$to be smaller did not modify our results significantly. The unknown $B(1)$ and $B(L e)$ defined by (4.14) will follow as byproducts once (4.9)-(4.13) are solved. Because $B(1)$ and $B(L e)$ effectively represent the apparent shifts in temperature and reactant mass fraction which the flame located at $|r| \ll \ell_{h}$ can feel, a two-term matching between (4.14) and (3.7) will yield $\delta y_{\infty}$ and $\delta T_{\infty}$ in terms of $r_{F}$, thereby enabling one to close (3.3) and (3.4).

Concerning the velocity field $\boldsymbol{V}(\boldsymbol{R})$ one must note that the condition $V(\infty)=-e_{x} V_{D}$ implied by (2.8) is not accurate enough a boundary condition at $R \gg 1$ in the limit $\ell_{h} / r_{Z} \rightarrow \infty$, due to the presence of a wake (figure 1) which renders the limit non-uniform. As shown by Kurdyumov and Liñàn (1999), a special treatment is needed to handle the wake and the potential flow surrounding it when $R \gg 1$. Following their analysis we introduce spherical coordinates $\dagger$ $(\vartheta, R)$ whose axis coincides with $e_{x}$ (figure 1 ), and a stream function $\psi$ such that

$$
V_{R}=\frac{1}{R^{2} \sin \vartheta} \frac{\partial \psi}{\partial \vartheta} \quad V_{\vartheta}=\frac{-1}{R \sin \vartheta} \frac{\partial \psi}{\partial R}
$$

where $V_{R}$ and $V_{\vartheta}$ represent the radial and the azimuthal components of $V$, respectively. The wake corresponds to $R \gg 1$ and to $\pi-\vartheta=\mathrm{O}\left(R^{-1 / 2}\right)$. Following the aforementioned work one seeks the solution profiles inside the wake in the self-similar form

$$
\psi=R F(\zeta) \quad \Theta=R^{-1} \Gamma(\zeta)
$$

with $\zeta \equiv R^{1 / 2}(\pi-\vartheta)$. To leading order in the limit $R \rightarrow \infty, \zeta$ fixed, the unknown functions $F(\cdot), \Gamma(\cdot)$ satisfy

$$
\begin{aligned}
& \operatorname{Pr}\left(\left(\frac{1}{\zeta} F^{\prime}\right)^{\prime \prime}+\frac{1}{\zeta}\left(\frac{1}{\zeta} F^{\prime}\right)^{\prime}\right)+\Gamma=\frac{F}{\zeta}\left(\frac{1}{\zeta} F^{\prime}\right)^{\prime} \\
& \zeta \Gamma^{\prime}=F \Gamma
\end{aligned}
$$

for $\zeta>0$, with $(\cdot)^{\prime} \equiv \mathrm{d}(\cdot) / \mathrm{d} \zeta . F$ and $\Gamma$ ought to fulfil the boundary conditions

$$
\begin{array}{ll}
F=0=\left(\frac{1}{\zeta} F^{\prime}\right)^{\prime} & \text { at } \quad \zeta=0 \\
F+\frac{1}{2} V_{D}^{2} \zeta^{2} \rightarrow F_{\infty} & \Gamma=0 \quad \text { at } \zeta=\infty
\end{array}
$$

where $F_{\infty}$ is an as yet unknown constant, together with the integral constraint

$$
2 \pi \int_{0}^{\infty} F^{\prime} \Gamma \mathrm{d} \zeta=-1 .
$$

The latter states that the heat convected in the wake is what is released at $R=0$ by the flame-ball.

The resolution (numerical in general) of (4.17)-(4.19) determines $F_{\infty}$ as a function $F_{\infty}\left(V_{D}\right)$ of $\mathrm{Pr}$ and of the presumed $V_{D} \equiv v_{D} / v_{h}$. Outside the wake and for $R \gg 1$ the flow is potential and may be shown to satisfy (see, e.g., Schneider 1981)

$$
\psi=-\frac{1}{2} R^{2} V_{D} \sin ^{2} \vartheta+\frac{1}{2} R(1-\cos \vartheta) F_{\infty}\left(V_{D}\right)
$$

$\dagger$ The cyclic coordinate is omitted in the following, owing to the axial symmetry of the process at hand. 
To solve equations $(4.9)-\left(4.14^{\prime}\right)$ for $0 \leqslant \vartheta \leqslant \pi$ we formulated them in terms of the stream function $\psi$ and vorticity. In addition to the vanishing of $\Theta, Y$ and vorticity, the above expression of $\psi$ was that used as the boundary condition at the surface of a large sphere centred at $R=0$ (with a radius $R_{+}$of a few $10^{2}$, typically) except in a small angular sector $\left(\pi-\vartheta \leqslant \alpha / R_{+}^{1 / 2}\right.$, $\alpha=\mathrm{O}(8)$ ) containing the wake; there, Neumann conditions on $\Theta, Y, \partial \psi / \partial R$ and vorticity were employed instead. For each presumed $V_{D}$, equations (4.6)-(4.14) could then be solved numerically; after use of $\log R$ as a radial coordinate in lieu of $R$ to concentrate nodes about $R=0$, the method combined three-point central finite differences and an implicit pseudo-time approach as to achieve convergence to the steady solutions. One may note that the finitedifference integration again computes the wake structure, which might seem unnecessary at first glance since it was already known from (4.17)-(4.19). In addition to avoiding any mismatch between wake and surroundings, this strategy has the virtue of borrowing the asymptotic stream function (4.20) from an independent calculation (equations (4.16)-(4.19)); this allows us to suppress the spurious, $R_{+}$-dependent large-scale vortices that would appear if the uniform-flow stream function $\left(-V_{D} \sin ^{2} \vartheta R^{2} / 2\right)$ had been used at $R=R_{+}$instead of (4.20).

\subsection{Main results from the numerics}

As shown in figure 2, only one $V_{D}$ allows the condition $V(\mathbf{0})=\mathbf{0}$ to be met. For the Prandtl number $\operatorname{Pr}=0.72$ we selected here, this scaled drift velocity is

$$
V_{D} \cong 0.432 \ldots
$$

and the value of $F_{\infty}$ in (4.18) and (4.20) is then 2.49; the associated temperature and radial velocity profiles across the wake are plotted in figure 3 .

The corresponding streamlines and the velocity along the symmetry axis are shown in figures 4 and 5, respectively: $V \cdot e_{x}$ is everywhere negative along the symmetry axis, except at $R=0$ where it vanishes. For future reference one notes that, once the condition $V(0)=0$ is fulfilled, the approximation

$$
\psi=-\frac{R^{3} \sin ^{2} \vartheta}{32 \pi P r}+\mathrm{O}\left(R^{4}\right)
$$

is found by a coordinate expansion to hold about $R=0$, whereby the velocity components locally read

$$
V_{R}=-\frac{R \cos \vartheta}{16 \pi P r}+\mathrm{o}(R) \quad V_{\vartheta}=\frac{3 R \sin \vartheta}{32 \pi P r}+\mathrm{o}(R)
$$

Actually, this behaviour extends rather far (figure 5).

Processing the $\Theta$ profile near the origin of far-field coordinates gave the pure number $B$ (1) defined in (4.14), namely $B(1)=-0.54 \times 10^{-2}$ for $\operatorname{Pr}=0.72$. Doing the same with $Y(R \rightarrow 0)$ yielded $B(L e)$, and repeating this for different $L e$ led to the results plotted as symbols in figure 6 . On the basis of arguments summarized in the appendix, we suggested that

$$
B(L e) \cong B(1) L e^{2}(k+(1-k) L e)^{-1 / 2}
$$

could constitute a satisfactory interpolation if the free parameter $k>0$ is suitably selected; as shown in figure 6 this is quite true, indeed, provided $k=0.34$ for $\operatorname{Pr}=0.72$. It is important to notice that $|B(L e)| / L e<|B(1)|$ if $L e<1$, implying a stronger influence of convection on temperature than on the mass fraction of a highly mobile fuel, as it should be. 


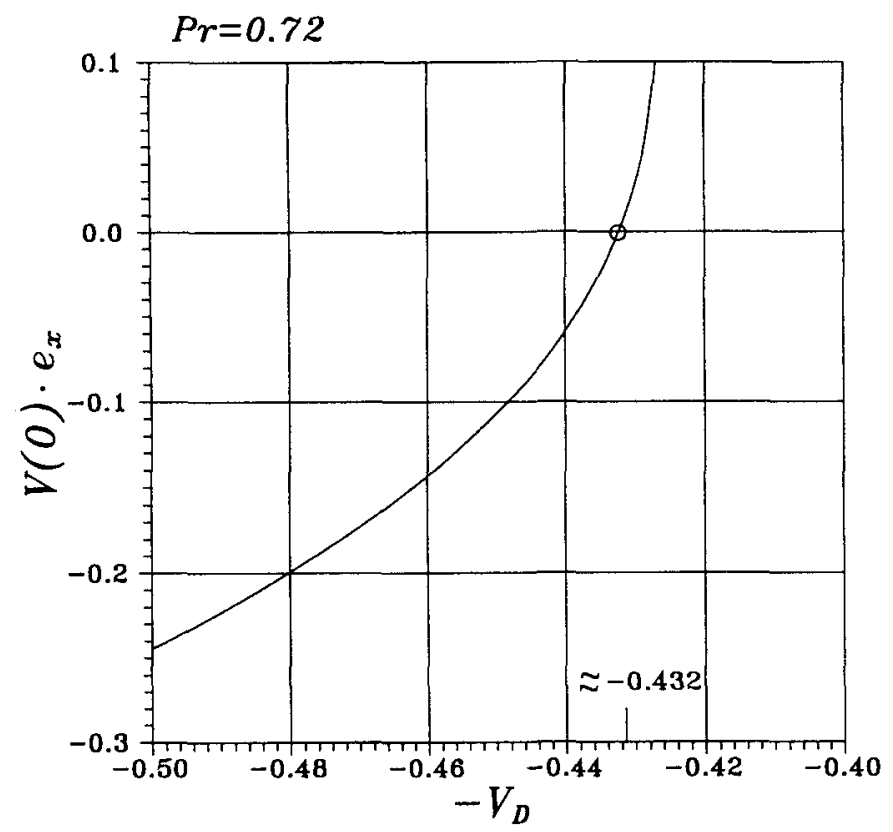

Figure 2. Only one value $\left(V_{D}=0.432\right)$ of $-\left.V \cdot e_{x}\right|_{R=\infty}$ leads to $\left.V \cdot e_{x}\right|_{R=0, \vartheta=0}=0$.

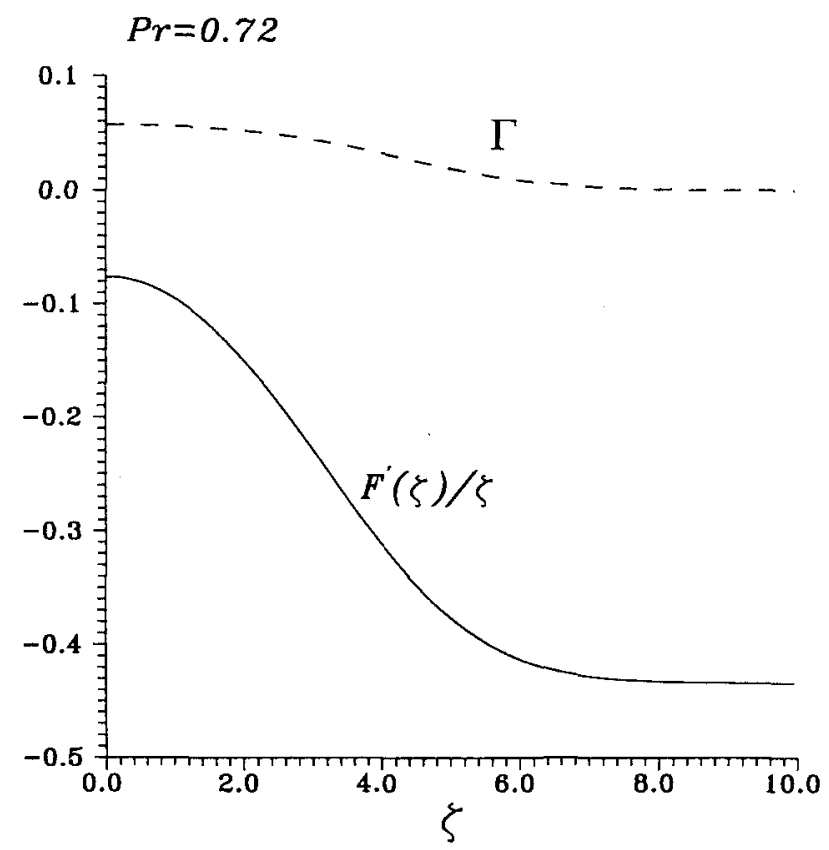

Figure 3. Scaled temperature $(\Gamma(\zeta))$ and velocity along the $e_{x}$-axis $\left(F^{\prime}(\zeta) / \zeta\right)$ in the wake as functions of the similarity variable $\zeta=(\pi-\vartheta) R^{1 / 2}$. The actual $T-T_{u}$ is proportional to $\Gamma(\zeta) / R$. 


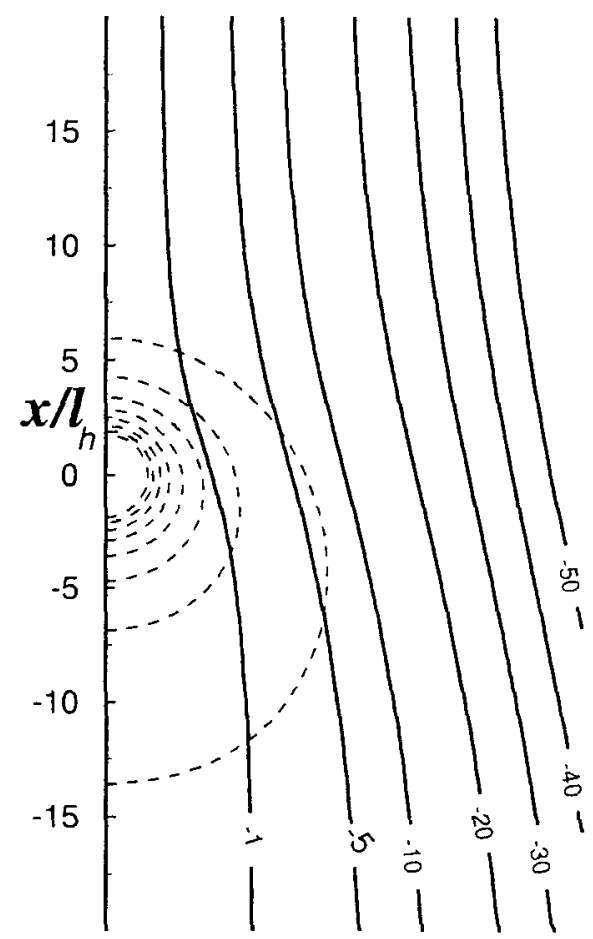

Figure 4. Streamlines (shown as full curves) in the hydrodynamical far field, as obtained numerically at the far-field scale. Each is labelled by the value of the stream function $\psi$. The broken curves are isotherms.

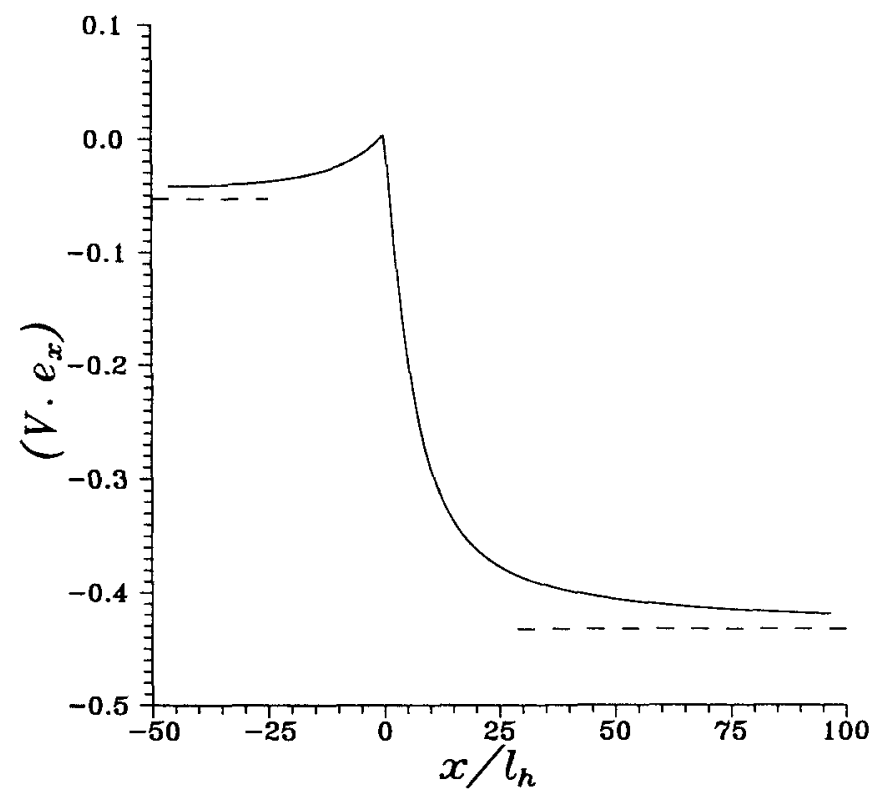

Figure 5. Velocity profile $V \cdot e_{x}$ along the axis of symmetry versus $R \cdot e_{x}$ for $V_{D}=0.432$. 


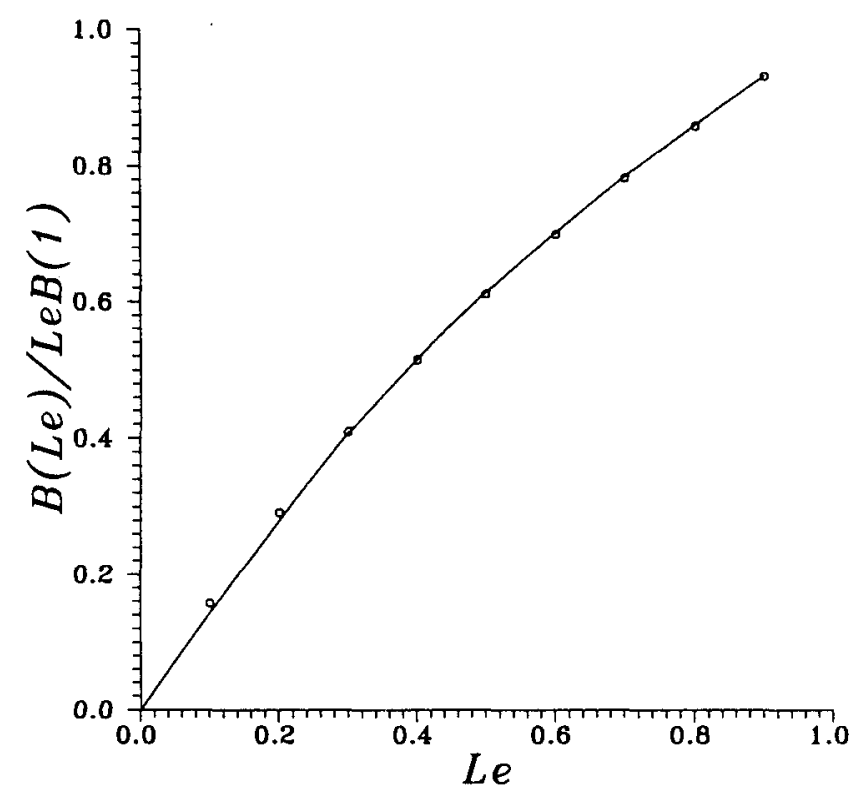

Figure 6. The function $B(L e) / L e B(1)$ as deduced from the numerical integration of $(2.1)-(2.8)$ for $\operatorname{Pr}=0.72$ (symbols). The full curve represents $L e /(k+(1-k) L e)^{1 / 2}$ with $k=0.34$.

\section{Flame-ball radius and drift velocity}

A two-term matching between (4.14) and (3.7) readily yields

$$
\begin{aligned}
& \delta T_{\infty}=B(1) \frac{4 \pi r_{F}}{D_{t h}}\left(T_{*}-T_{u}\right)\left(4 \pi r_{F}\left(\frac{T_{*}-T_{u}}{T_{u}}\right) g\right)^{1 / 2}<0 \\
& \delta y_{\infty}=-\frac{B(L e)}{L e} \frac{4 \pi r_{F}}{D_{t h}} y_{u}\left(4 \pi r_{F}\left(\frac{T_{*}-T_{u}}{T_{u}}\right) g\right)^{1 / 2}>0
\end{aligned}
$$

once the definitions (4.2) and (4.3) of $\left(T_{h}-T_{u}\right)$ and $\left(y_{h}-y_{u}\right)$ are made use of, whereby (3.3) leads to

$$
\frac{\delta T_{R}}{T_{*}}=\left(B(1)-\frac{B(L e)}{L e}\right) \frac{4 \pi r_{F}}{D_{t h}} \frac{T_{*}-T_{u}}{T_{*}}\left(4 \pi r_{F}\left(\frac{T_{*}-T}{T_{u}}\right) g\right)^{1 / 2} .
$$

One may note that $\delta T_{R}$ is negative if $L e<1$, and is isotropic (i.e. angle independent) to leading order, despite the anisotropic flow field surrounding the flame-ball; this can be traced back $\dagger$ to the fact that $|V(R)|$ is bounded by $\mathrm{O}(R)$ as $R \rightarrow 0$ (see equation (4.23)). Requiring $\delta T_{R} / T_{*}$ to be $\mathrm{O}(1 / Z e)$, so as to have $r_{F}=\mathrm{O}\left(r_{Z}\right)$, led to the anticipated distinguished limit (3.5).

It is now a simple matter to combine (3.4) and (5.2) into a closed equation for the scaled flame-ball radius $\mathfrak{R} \equiv r_{F} / r_{Z}$, namely

$$
\mathfrak{R}=\exp \left(G^{1 / 2} \mathfrak{R}^{3 / 2}\right)
$$

in which the scaled Grashof number $G$ reads as

$$
G=\left(Z e 2 \pi b(L e) \frac{r_{Z}}{D_{t h}}\right)^{2} 4 \pi r_{Z} \frac{T_{*}-T_{u}}{T_{u}} g
$$

$\dagger$ Private communication from J Velazquez and M Herrero, Complutense Univ., Madrid. 


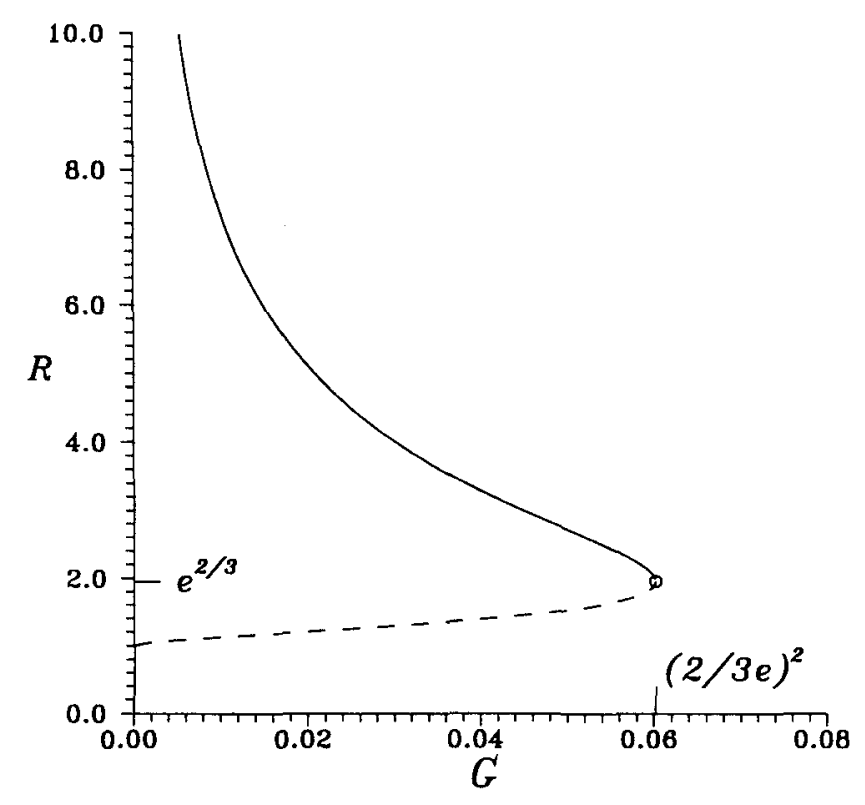

Figure 7. Reduced flame-ball radius $\Re=r_{F} / r_{Z}$ versus the Grashof number $G$ defined by equation (5.4).

and measures the intensity of gravity; in (5.4), $Z e \equiv T_{a}\left(T_{*}-T_{u}\right) / T_{*}^{2}$ and the positive function $b(L e) \equiv-B(1)(1-B(L e) / L e B(1))$ is available, e.g. from (4.24) or figure 6.

The predicted $(\Re, G)$ curve is plotted in figure 7 . It resembles what was obtained when studying the influence of volumetric losses $\left(\delta T_{R} \sim-\mathfrak{R}^{2}\right.$ or $\sim-\mathfrak{R}$; Buckmaster et al $1990,1991)$ or prescribed ambient velocity gradients $\left(\delta T_{R} \sim-\mathfrak{R}\right.$, Buckmaster and Joulin 1991). In mathematical terms this is so because the reaction-temperature drop again increases algebraically with $\Re$, whereas (3.4) involves an exponential (any rapidly varying convex function of $\delta T_{R}$ would also do). In fact, both (5.3) and the response to prescribed velocity gradients may be written as $\Re=\exp (|\gamma| \Re)$, where $\gamma^{2}$ measures the actual velocity gradients experienced by the flame-ball.

Here, steady solutions exist only when $G \leqslant G_{\text {crit }}=(2 / 3 e)^{2}$, and the value $\mathfrak{R}_{\text {crit }}$ of $\Re$ associated with $G_{\text {crit }}$ is $e^{2 / 3}$. The branch $\Re<\Re_{\text {crit }}$ is believed to be unstable, since the Zel'dovich solution $\Re=1$ is, and because the turning point at $\left(\Re_{\text {crit }}, G_{\text {crit }}\right)$ is compatible with marginal stability: for $G \rightarrow G_{c r i t}-0$ the then infinitesimal difference between the two steady solution profiles is a solution to the linearized conservation equations and provides one with a neutral eigenmode. A proof that the steady solutions corresponding to $\mathfrak{R}>\mathfrak{R}_{\text {crit }}\left(\mathfrak{R}<\mathfrak{R}_{\text {crit }}\right.$ ) are stable (unstable) against small changes in $\mathfrak{R}$ would require solving a linearized version of (4.9)-(4.14') at the hydrodynamical scale $r=\mathrm{O}\left(\ell_{h}\right)$, because such processes are known (Buckmaster et al 1990, 1991, Joulin 1985) to spontaneously evolve on the time scale $t=\mathrm{O}\left(Z e^{2} r_{F}^{2} / D_{t h}\right)=\mathrm{O}\left(\ell_{h}^{2} / D_{t h}\right)$. Even though the associated flame-ball evolution is quasi-steady, the resulting full coupling between unsteadiness and free convection in the far field to date precluded any resolution of this aspect of the stability problem.

Concerning the flame-ball stability against deformations of the reactive layer, the situation is likely to be simpler. Indeed, the adequate time scale is then $t=\mathrm{O}\left(Z e r_{F}^{2} / D_{t h}\right)$. 
Accordingly, the temperature and mass-fraction disturbances have an $\mathrm{O}\left(r_{F} Z e^{1 / 2}\right)$ range, which is asymptotically intermediate between $r_{F}$ and $\ell_{h}=\mathrm{O}\left(Z e r_{F}\right)$ and allows us to employ (4.23) to describe the unperturbed flow field locally. The resulting mathematical stability problem is then analogous to that encountered when handling flame-balls subjected to velocity gradients (Buckmaster and Joulin 1991), where the unperturbed velocity field had no direct influence on the growth/decay rates (only an indirect one, via the steady value of $\mathfrak{R}$ ). One may thus surmise that no three-dimensional flame-ball instability will show up here, because no volumetric heat-loss term acts inside the flame-ball (see the above references).

At criticality, $g=g_{\text {crit }}$ satisfies

$$
\frac{g_{c r i t} r_{Z}^{3}}{D_{t h}^{2}} Z e^{2} \frac{T_{*}-T_{u}}{T_{u}}=\frac{4}{9 e^{2} 16 \pi^{3} b^{2}(L e)}
$$

to be compared with (3.5). For $T_{*}=5 T_{u}, Z e=10, L e=0.3\left(b(L e) \cong 3 \times 10^{-3}\right)$, $D_{t h}=10^{-5} \mathrm{~m}^{2} \mathrm{~s}^{-1}$ and $\dagger r_{Z}=10^{-3} \mathrm{~m}$ this is $g_{\text {crit }}=3.4 \times 10^{-3} \mathrm{~m} \mathrm{~s}^{-2}$, a value to be discussed later.

As for the drift velocity $v_{D}$, the definitions (4.2) and (4.6) of $v_{h}$ and $V$ imply

$$
v_{D}=V_{D}\left(g 4 \pi r_{Z}\left(\frac{T_{*}-T_{u}}{T_{u}}\right)\right)^{1 / 2} \mathfrak{R}^{1 / 2}=V_{D}\left(g 4 \pi r_{F}\left(\frac{T_{*}-T_{u}}{T_{u}}\right)\right)^{1 / 2} \quad V_{D}=0.432
$$

Such a $\left(v_{D}, r_{F}\right)$ relationship is similar to that proposed by Ronney et al (1998).

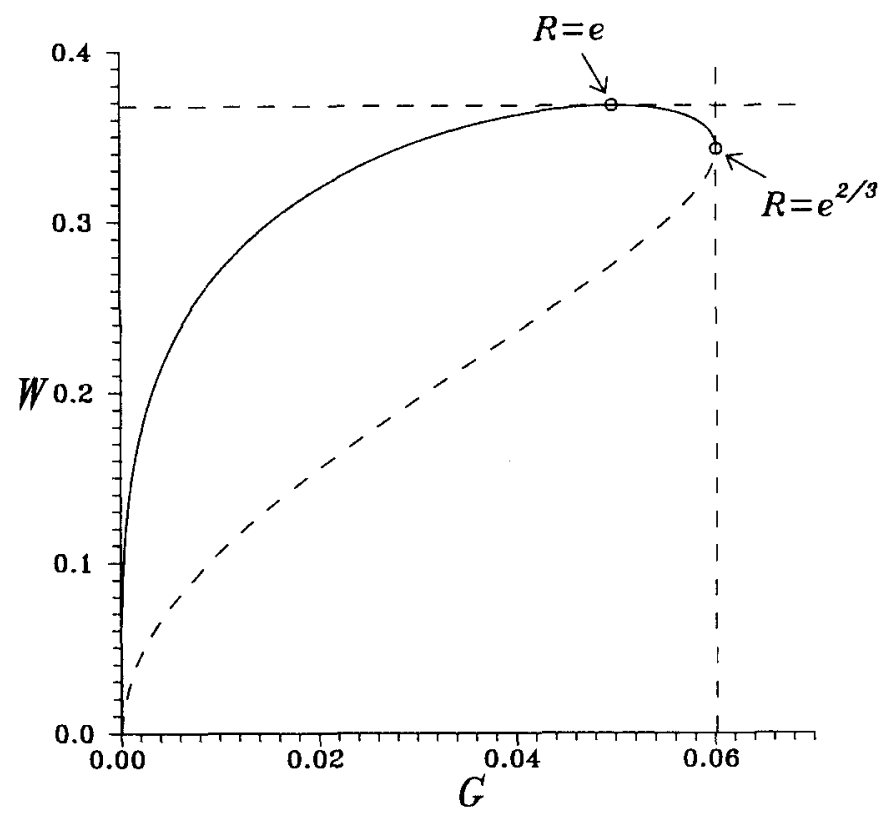

Figure 8. Variations of the reduced drift velocity $W$ (equation (5.8)) with scaled gravity $G$ (equation (5.4)), as implied by (5.7).

\footnotetext{
$\dagger$ In the absence of gravity, but with volumetric heat losses included (Buckmaster et al 1990, 1991) $r_{F}$ should exceed $r_{F} e^{1 / 2}$ for stability reasons, and $r_{Z}$ may be noticeably smaller than the observed $r_{F}(0.5 \mathrm{~cm}$, typically); hence our guess on $r_{Z}$.
} 
Once combined, equations (5.3) and (5.6) yield a drift velocity versus gravity curve in parametric form

$$
W=(\log \mathfrak{R}) / \mathfrak{R} \quad G=(\log \mathfrak{R})^{2} / \mathfrak{R}^{3}
$$

in which the Peclet number $W$ reads as

$$
W=\frac{v_{D} r_{Z}}{D_{t h}} Z e \frac{2 \pi b(L e)}{V_{D}} \quad V_{D}=0.432 .
$$

$W$ is plotted as a function of $G$ in figure 8 ; it is seen to have a maximum $W_{m}=e^{-1}$ when $\mathfrak{R}=\mathfrak{R}_{m}=e>e^{2 / 3}$ and $G=G_{m}=e^{-3}<(2 / 3 e)^{2}$. For $T_{*}=5 T_{u}, Z e=10, L e=0.3$ $\left(b(L e) \cong 3 \times 10^{-3}\right), D_{t h}=10^{-5} \mathrm{~m}^{2} \mathrm{~s}^{-1}$ and $r_{Z}=10^{-3} \mathrm{~m}$, one has $v_{m} \equiv \max \left(v_{D}\right)$ $=8 \times 10^{-3} \mathrm{~m} \mathrm{~s}^{-1}$, a value also to be discussed later.

\section{Discussion}

To close the above analysis we explicitly made use of (2.8), i.e. that the flame-ball would be dragged by the flow it itself generates in the presence of gravity. As aptly pointed out recently by Buckmaster and Ronney (1999), a flame-ball subjected to an ambient gradient of $(T+Q y / c L e)$ acquires a propagation velocity $\left(v_{F}\right.$, say) relative to the gas, which is given by

$$
v_{F} \sim \frac{D_{t h}}{T_{*}} \nabla(T+Q y / c L e)
$$

at least if small enough. In the present situation, the gradient of $(T+Q y / c L e)$ results from the outer, free-convection field and from the different ways temperature and reactant mass fraction react to it. The gradient involved in (6.1) may then be estimated (from equation (4.14)) as $\mathrm{O}\left(\left(T_{h}-T_{u}\right) / \ell_{h}\right)$, leading to

$$
\frac{v_{F}}{v_{D}} \sim \frac{T_{h}-T_{u}}{T_{*}} \sim \frac{\log \Re}{Z e}
$$

which is negligible as $Z e \rightarrow \infty$ whenever $\log \mathfrak{R}=\mathrm{O}(1)$, as is assumed here. Even if $v_{F}$ is negligible, the flame-ball might rise relative to its immediate environment (in the same way as a gas bubble does in a denser liquid), because the pressure gradients induced at the $r=\mathrm{O}\left(r_{F}\right)$ scale by buoyancy and $\mathrm{O}(1)$ density changes need be compensated. The resulting 'Archimedean' velocity, $v_{A}$ say, caused by local buoyancy can be estimated from the lowReynolds-number approximation of (2.5). This yields $\left(\rho-\rho_{u}\right) g \sim \mu_{u} v_{A} / r_{F}^{2}$, then

$$
\frac{v_{A}}{v_{D}} \approx \frac{G \mathfrak{R}^{3}}{Z e^{2}} \frac{\ell_{h}}{r_{F}} \approx \frac{\log \Re}{Z e}
$$

once (5.3) has been made use of. Accordingly $v_{A} \sim v_{F} \ll v_{D}$, equation (2.8) was legitimate and our analysis is self-consistent.

It is now appropriate to comment on the numerical values of $g_{c r i t}$ and $v_{m} \equiv \max \left(v_{D}\right)$ obtained in section 5 , which are very small indeed for $r_{Z} \approx 10^{-3} \mathrm{~m}$. Admittedly we assumed constant $\lambda$ and $\rho D$, whereas the heat conductivity of gases noticeably increases with $T$, so that our estimates might be too crude. However, one can show that employing a non-constant $\lambda(T)$ (Le being kept fixed) would not modify (3.4) and would merely endow the definitions (5.4) and (5.8) of $G$ and $W$ with the extra factors $\left(\bar{\lambda} / \lambda_{u}\right)^{3}$ and $\left(\bar{\lambda} / \lambda_{u}\right)$, respectively, where

$$
\bar{\lambda} \equiv \int_{T_{u}}^{T_{*}} \lambda(T) \mathrm{d} T /\left(T_{*}-T_{u}\right)
$$

is an average conductivity $\left(\bar{\lambda}>\lambda_{u}\right)$. Accordingly, $g_{c r i t}$ and $v_{m}$ would be still lower than previously estimated. 
The value $r_{Z} \sim 10^{-3} \mathrm{~m}$ we chose as a numerical example could be an overestimate, in which case $g_{\text {crit }} \sim r_{Z}^{-3}$ and $v_{m} \sim r_{Z}^{-1}$ would have been underestimated; unless this is true it is difficult to understand from the present self-consistent analysis how flame-balls of some sort could resist gravity fields that are not exceedingly small compared to the terrestrial one.

The above remarks suggest that our present analysis does not cover the entire response properties of localized combustion spots to gravity fields. This can even be detected from the above estimates. When $r_{F}\left(T_{h}-T_{u}\right) / T_{*} \ell_{h}$ becomes of the order of $Z e^{-1}$, i.e. for $\log \Re=\mathrm{O}\left(Z e^{1 / 2}\right)$, the variations in temperature, which the non-vanishing $\nabla(T+Q y / c L e)$ caused by natural convection induces along the reaction zone (where $y=0$ ), are strong enough to spoil the latter's spherical symmetry. In such circumstances $v_{F} / v_{D}$ and hence $v_{A} / v_{D}$ would still be small $\left(\mathrm{O}\left(Z e^{-1 / 2}\right)\right)$, yet only moderately small, unfortunately, for $Z e=\mathrm{O}(10)$; $v\left(r=\mathrm{O}\left(r_{F}\right)\right) r_{F} / D_{t h}=\mathrm{O}\left(Z e^{-1}\right)$ would be non-negligible, because convection would then induce yet another small asymmetry between $T-T_{u}$ and $\left(y-y_{u}\right) / L e$, thereby further affecting $\delta T_{R}$ and hence the flame shape. Scale separation would still hold $\left(r_{F}=\ell_{h}=\mathrm{O}\left(Z e^{-1 / 2}\right)\right)$, as well as (2.8), but the free-boundary problem at the $r=\mathrm{O}\left(r_{F}\right)$ scale is certainly rather difficult to tackle. Unfortunately, solving it is likely to be one of the necessary steps needed to understand how the regimes we have studied here can be continuously linked to those pertaining to near-limit flames at Earth gravity.

As another extension of the present analysis, one might also think of alluding to radiant volumetric heat losses, which will tend to lower $T_{R}$ (thereby increasing $r_{F}$ and its equivalent source strength $q$ ) and if they happen to have a range comparable to $\ell_{h}$, these will also shorten the effective range of thermal effects (hence lowering the overall influence of gravity at fixed $q$ ). This competition is the subject of current works, which are undoubtedly prerequisites before any serious comparison with experiments is attempted, especially in near-limit conditions. In such situations chemical-kinetic effects would also be important and the scale-separation $\ell_{h} \gg r_{F}$ for $Z e \gg 1$ will greatly help incorporate them: the flame-ball structure corresponding to $r_{F}=\mathrm{O}\left(r_{Z}\right)$ can indeed be worked out independently of the hydrodynamical problem, even if a detailed chemical scheme is adopted.

\section{Acknowledgments}

GJ is indebted to the Universitad Politecnica (UP) de Madrid (ETS Ingenieros Aeronauticos) for hospitality and to IBERDROLA for financial support, during two two-month stays when this work was completed; special thanks also go from GJ to Dr R Blouquin, and to Professors F J Higuera and A Liñàn for help and unabated kindness. The work of VNK was supported by the DGICYT post-doctoral fellowship at UP of Madrid, and by INTA under contract INTA-UPM Mezcla y Combustión. The constructive comments by Professor F A Williams (California University) were also appreciated.

\section{Appendix A. On the choice of formula (4.24)}

Using $\boldsymbol{R}=\boldsymbol{\xi} / \sqrt{L e}$ and $Y=L e^{2 / 3} \Psi$ in equations (4.11)-(4.14) converts them into

$$
\begin{aligned}
& \sqrt{L e} V(\xi / \sqrt{L e}, \vartheta) \cdot \nabla_{\xi} \Psi=\nabla_{\xi}^{2} \Psi \\
& \Psi(\infty, \vartheta)=0 \quad \Psi(\xi \rightarrow 0, \vartheta)=\frac{1}{4 \pi \xi}+\frac{B(L e)}{L e^{3 / 2}}+\cdots
\end{aligned}
$$

The limit $L e \rightarrow \infty$ allows one to employ the local form (4.23) of $V$, whereby (A.1) and its solution subject to $4 \pi \xi \Psi(\xi \rightarrow 0, \vartheta)=1$ become parameter free; therefore $B(L e) / L e^{3 / 2}$ should then be a pure number, and $B(L e \rightarrow \infty) \sim L e^{3 / 2}$. Actually (4.23) holds over a rather 
wide zone (figure 5), suggesting that the above asymptotic behaviour of $B(L e)$ could be valid down to moderate arguments, hopefully for $L e<1$.

If $L e \rightarrow 0$, rewriting (4.11)-(4.14) and $Y$ as

$$
\begin{aligned}
& Y=\operatorname{Le} \varphi \quad \nabla^{2} \varphi=\operatorname{LeV} \cdot \nabla \varphi \\
& \varphi(R \rightarrow 0)=\frac{1}{4 \pi R}+\frac{B(L e)}{L e}+\cdots \quad \varphi(\infty)=0
\end{aligned}
$$

shows that $B(L e) / L e$, which results from convection and hence from the right-hand side of the balance for $\varphi$, should be $\mathrm{O}(L e)$ as $L e \ll 1$; hence $B(L e \rightarrow 0) \sim L e^{2}$.

Equation (4.24) is compatible with the above limiting behaviours and is correct for $L e=1$. $k=0.34$ for $\operatorname{Pr}=0.72$ was selected to ensure a best fit. 